\title{
Nanomolar concentration of blood-soluble drag-reducing polymer inhibits experimental metastasis of human breast cancer cells
}

This article was published in the following Dove Press journal:

Breast Cancer - Targets and Therapy

24 February 2017

Number of times this article has been viewed

\author{
Zhijie Dingl,* \\ Marion Joy,* \\ Marina $\bigvee$ Kameneva ${ }^{1-3}$ \\ Partha Roy ${ }^{1,3-6}$ \\ 'Department of Bioengineering, \\ ${ }^{2}$ Department of Surgery, ${ }^{3}$ McGowan \\ Institute of Regenerative \\ Medicine, ${ }^{4}$ Department of Pathology, \\ ${ }^{5}$ Department of Cell Biology, \\ 'Magee Women's Research Institute, \\ University of Pittsburgh, Pittsburgh, \\ PA, USA \\ *These authors contributed equally to \\ this work
}

\begin{abstract}
Metastasis is the leading cause of cancer mortality. Extravasation of cancer cells is a critical step of metastasis. We report a novel proof-of-concept study that investigated whether non-toxic blood-soluble chemical agents capable of rheological modification of the near-vessel-wall blood flow can reduce extravasation of tumor cells and subsequent development of metastasis. Using an experimental metastasis model, we demonstrated that systemic administration of nanomolar concentrations of so-called drag-reducing polymer dramatically impeded extravasation and development of pulmonary metastasis of breast cancer cells in mice. This is the first proof-of-principle study to directly demonstrate physical/rheological, as opposed to chemical, way to prevent cancer cells from extravasation and developing metastasis and, thus, it opens the possibility of a new direction of adjuvant interventional approach in cancer. Keywords: breast cancer, metastasis, extravasation, hemodynamics, drag-reducing polymer, blood cell traffic, microvessels
\end{abstract}

\section{Introduction}

Metastasis of tumor cells is the primary cause of cancer-associated mortality. To fully metastasize, cancer cells undertake a sequence of discrete steps: stromal invasion, intravasation into systemic circulation, survival in the circulation, arrest at certain areas in vasculature, and extravasation followed by survival and proliferation at the ectopic site. It is being increasingly recognized that arrest in vasculature and subsequent extravasation of tumor cells require their interactions with a number of host cells including vascular endothelial cells (VEC), platelets, lymphocytes, macrophages, mast cells, fibroblasts, and bone-marrow-derived progenitor cells, ${ }^{1}$ and these interactions are mediated by various cell adhesion molecules. Hemodynamics in microvessels has also been shown to be involved in the process of adhesion of circulating tumor cells (CTC) to VEC (via binding to neutrophils), and their subsequent extravasation. ${ }^{2}$ Near vessel wall hemodynamic parameters such as shear stress and shear rate, which are defined by local blood viscosity and velocity, were suggested to have a strong effect on the transport of tumor cells modulating cell-cell collisions and cell-cell contact time. Slow blood flow near vessel wall increases probability of tumor cells to attach to neutrophils and thus VEC, while an increase in flow rate leads to a reduction in the interaction time during cell collisions. ${ }^{3-5}$ Shear stress also impacts surface expression of adhesion molecules in VEC., 7 Given the importance of hemodynamic parameters in regulating cell-cell adhesion, we speculated that hemodynamic-alteration strategy could be a novel way to restrain tumor cell extravasation and subsequent development of metastasis.
Correspondence: Partha Roy Department of Bioengineering, University of Pittsburgh, 306 Center for Biotechnology and Bioengineering 300 Technology Drive, Pittsburgh, PA 15219, USA

Tel +l 4I 26247867

Fax + I 4123838788

Email par19@pitt.edu 
In microvessels (below $\sim 300 \mu \mathrm{m}$ diameter), there is a tendency of red blood cells (RBCs) to move toward the center of the blood vessel creating a near-wall plasma layer, a phenomenon known as Fåhraeus effect. ${ }^{8}$ This phenomenon (reduced near-wall concentration of RBCs) along with a low near-wall flow velocity creates an auspicious environment for neutrophils to cargo tumor cells through the vessel wall. Nanomolar intravascular concentrations of blood-soluble long-chain polymers (so-called drag-reducing polymers or DRPs) reduce or eliminate cell-free layer near the vessel wall in the microcirculation (Figure 1) and significantly increase the traffic of RBCs in small vessels and capillaries. ${ }^{9}$ Moreover, these polymers modulate blood flow velocity profile increasing near-wall velocity gradient and shear forces. ${ }^{10}$ These polymers were already proven to reduce platelet margination in microchannels in vitro ${ }^{11}$ and to diminish inflammatory reaction in animals implanted with biodegradable scaffolds presumably via reduction of near-wall leukocyte concentrations. ${ }^{12}$ Because of the ability of DRPs to modify near-wall flow dynamics, we conducted a proof-of-principle study to test a novel hypothesis whether systemic administration of DRP impairs extravasation and therefore development of metastasis of breast cancer cells.

\section{Materials and methods}

\section{Preparation of DRP solution}

Polyethylene oxide (PEO [4000 kDa MW] - Sigma-Aldrich, St. Louis, MO, USA), a high molecular weight synthetic DRP, was dissolved in sterile saline at a concentration of $0.1 \%$ and then dialyzed against saline for $24 \mathrm{~h}$ using a 50-kDa MW cutoff membrane (regenerated cellulose membrane; Spectra/Por; Spectrum Laboratories, Inc., Rancho Dominguez, CA, USA) to remove low molecular weight polymer fractions. To confirm complete dissolution and rule out mechanical degradation of the polymer during preparation, viscosity of the polymer solution was measured using capillary viscometers (Cannon Instrument, Co., State College, PA, USA). PEO solution was

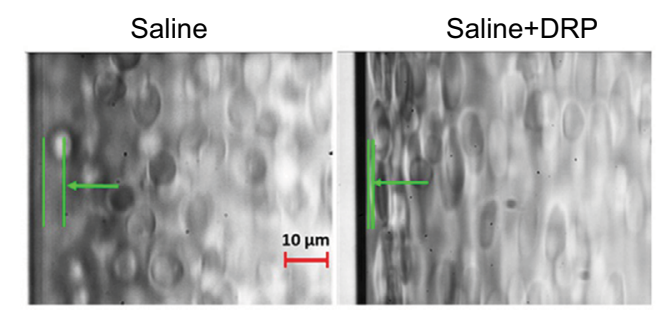

Figure I Effect of drag-reducing polymer (DRP) on cell-free layer near vessel wall. Notes: Microscopic images of red blood cell (RBC) suspensions flowing in a straight glass rectangular microchannel $(0.1 \times 0.1 \times 25 \mathrm{~mm}$, flow rate $=0.3 \mathrm{~mL} / \mathrm{min}): \mathrm{RBCs}$ were suspended in saline with or without DRP at a concentration of $\sim 10 \mathrm{ppm}$. Cell-free layer is the distance between the microchannel wall and the indicated arrow. Note that the RBCs on the right (saline+DRP) image are more elongated than RBCs suspended in saline (on the left) since they are exposed to the higher shear stress due to a closer location to the wall (scale bar: $10 \mu \mathrm{m}$ ). finally diluted with saline to the concentration of $50 \mathrm{ppm}$ and sterilized using a $0.22-\mu \mathrm{m}$ filter before injecting into mice. Fresh solution was prepared before each animal test.

\section{Cell culture}

Generation and culture of MDA-MB-231 (MDA-231) breast cancer cell line (source: ATCC, Manassas, VA, USA) stably expressing red fluorescence protein (RFP) has been previously described. ${ }^{13}$

\section{Animal experiments}

DRP solution (50 ppm in a 30-50 $\mu \mathrm{L}$ ) or equivalent volume of saline was injected into the lateral tail vein of female athymic nude mice ( $\sim 25 \mathrm{~g}$ weight). The animals were subsequently injected with $1 \times 10^{6}$ RFP-expressing MDA-231 cells suspended in $50 \mu \mathrm{L}$ sterile phosphate-buffered saline (PBS) through the lateral tail-vein route. In some experiments, two additional rounds of DRP or sterile saline injections were performed over the course of the experiment before the animals were sacrificed. Lungs were harvested, fixed, and surface-imaged by a wide-field fluorescence microscope to detect RFP-positive tumor cells as we had done before. ${ }^{14}$ The institutional Animal Care Committee of the University of Pittsburgh reviewed and approved this study, and all animal experiments were performed according to the approved protocol and followed relevant institutional and national guidelines of humane animal care act (Animal Welfare Act, 7 USC 2131 et seq).

\section{Results and discussion}

To determine whether systemic administration of DRP can have an effect on tumor cell extravasation, we performed an experimental metastasis assay where we injected MDA-231 breast cancer cells stably expressing RFP through the lateral tail vein of immunodeficient mice and examined lungs for tumor cell presence $24 \mathrm{~h}$ after intravenous (IV) injection. Although a large number of tumor cells (1-2 millions) are typically injected into this in vivo model, the vast majority of circulating cancer cells is destroyed in the microvasculature. This is thought to be due to excessive physical deformation from shear stresses and/or possibly other cytocidal mechanisms. ${ }^{15-17}$ Only a very small percentage of these surviving cells eventually manage to extravasate into the tissue parenchyma. In fact, hemodynamic destruction of circulating cancer cells is considered to be an important underlying cause of the "metastatic inefficiency". Our initial characterization of MDA-231 cells in this tail-vein injection model showed that at an early time point $(2 \mathrm{~h})$ after injection, MDA-231 cells are still restricted in the lung microvasculature, as evident from tumor cell distribution mimicking the characteristic 
honeycomb network of blood vessels on the lung surface (left panel of Figure 2A). The vast majority of these cells disappeared leaving only a sparse population of these cells in the lung bed at the $24 \mathrm{~h}$ time point (right panel of Figure 2A), and this distribution is consistent with our previous observation in a recently published study. ${ }^{14}$ In the actual experiments, lungs from control (saline-injected) mice also revealed this sparse distribution of MDA-231 cells $24 \mathrm{~h}$ after injection (left panel of Figure 2B). In stark contrast, those from DRP-treated mice at the $24 \mathrm{~h}$ time point exhibited distribution of tumor cells very much similar to the pattern seen in mice at early time point ( $2 \mathrm{~h}$ ) after injection (right panel of Figure $2 \mathrm{~B}$ ). Note that at the $2 \mathrm{~h}$ time point, lungs from saline/tumor cell and DRP/ tumor cell-injected animals appear indistinguishable from those injected from tumor cell (also in PBS) alone (left side

A Injection: tumor cell only
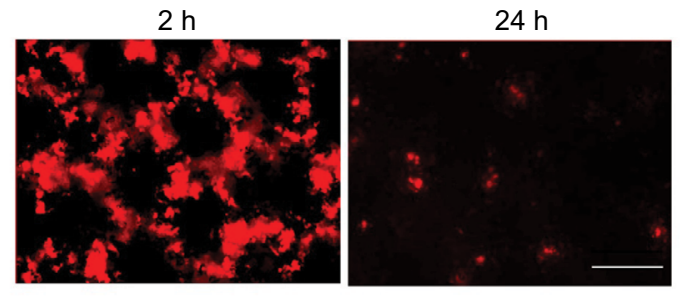

B

Injection: tumor cell+saline/DRP

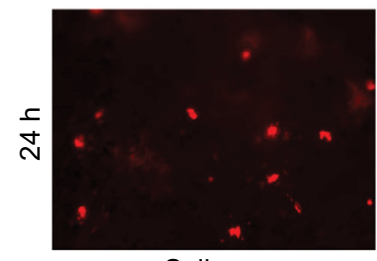

Saline

C

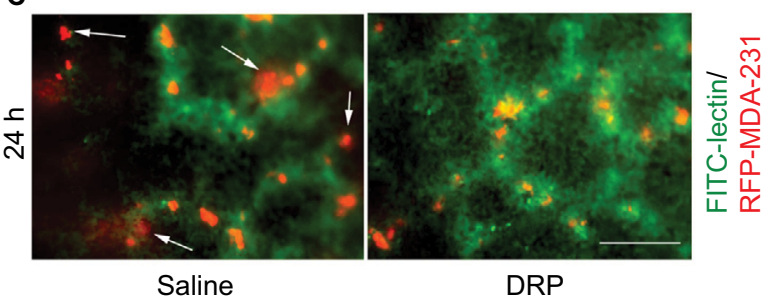

Figure 2 Effect of DRP on lung extravasation of MDA-23I cells.

Notes: (A and B) Representative fluorescence images (10X) of lungs of mice following tail-vein inoculation of RFP-expressing MDA-23I cells for the indicated end points of observation and co-injection conditions (drag-reducing polymer [DRP] or saline). Note that fluorescence differential between 2 and $24 \mathrm{~h}$ time points is a reflection of high versus low cell densities at these two time points. At the $2 \mathrm{~h}$ time point, large clusters of cells give rise to higher cumulative fluorescence. A sparse population of either individual or very small cluster of cells in the lungs gives an impression of lower fluorescence at the $24 \mathrm{~h}$ time point. It is also to be noted that not all cells are in focus in the wide-field fluorescence images, which account for cellto-cell variability of fluorescence in a given field. Panel (C) shows RFP-positive tumor cells within FITC-lectin-stained blood vessels in saline versus DRP-injected animals (FITC-lectin was injected $30 \mathrm{~min}$ prior to sacrificing the animals). Extravasated tumor cells in the control animals are indicated by arrows (data representative of three animals/group; scale bars: $100 \mu \mathrm{m}$ ).

Abbreviations: RFP, red fluorescence protein; FITC, fluorescein isothiocyanate. panel Figure 2A; data not shown). These data suggest that DRP promotes retention of tumor cells in the vasculature. To assess the effect of DRP on the extravasation of tumor cells, we stained the blood vessels by injecting fluorescein isothiocyanate (FITC)-lectin $30 \mathrm{~min}$ prior to sacrificing either control or DRP-treated animals at the $24 \mathrm{~h}$ time point. Control mice clearly showed evidence of extravasated RFP-positive tumor cells (marked by arrows) into the lung parenchyma (left panel of Figure 2C), while in DRP-treated animals RFP-positive tumor cells were mostly entrapped inside the FITC-labeled blood vessels (this is also indicated by yellowish appearance of tumor cells due to co-localization of green and red fluorescence; right panel of Figure 2C). These observations suggest that DRP inhibits the extravasation step of metastasis.

To further determine whether lateral tail vein administration of DRP affects development of pulmonary metastases of breast cancer cells, we performed similar experimental metastasis assay with MDA-231 cells except we assessed lung colonization of breast cancer cells 10-11 days after their injection through the lateral tail vein. In these experiments, a total of three rounds of IV injections of either PBS (vehicle control) or DRP were performed: day 0 (20 min before tumor cell injection), day 4 and day 8. Multiple rounds of DRP injection was performed because it has been shown that PEO molecules are mechanically degraded in the vascular system into shorter fragments, which are easily excreted by the urinary system with a mean retention time of $\sim 77 \mathrm{~h} .{ }^{18}$ Furthermore, several long-term animal studies demonstrated that IV injections of nanomolar concentrations of DRPs 2-3 times per week are sufficient to maintain the effective concentrations of DRP in the vascular system. ${ }^{12,19,20}$ Figure $3 \mathrm{~A}$ shows that control mice injected with sterile saline developed prominent lung metastases as evident from the large clusters of fluorescent cells. By contrast, lungs of DRP-treated mice showed presence of only few individual and tiny clusters of tumor cells. Quantification of data revealed a dramatic $\sim 7$-fold difference $(p<0.0001)$ in the average metastases readout between control and DRP-treated mice (Figure 3B). These data demonstrate that systemic administration of DRP can inhibit development of metastases from breast cancer cells in the circulation.

How DRPs inhibit extravasation of breast cancer cells? Extravasation of tumor cells in lungs can potentially involve blood vessels spanning a wide range of diameter. DRPs do not likely have any effect on extravasation within narrow capillaries $(<10 \mu \mathrm{m}$ in diameter) since in this case tumor cells completely occlude the blood vessel. However, in larger microvessels, DRPs can potentially impede the attachment of tumor cells to VEC and inhibit extravasation via reducing near-wall concentrations of tumor cells and/or other types of blood 

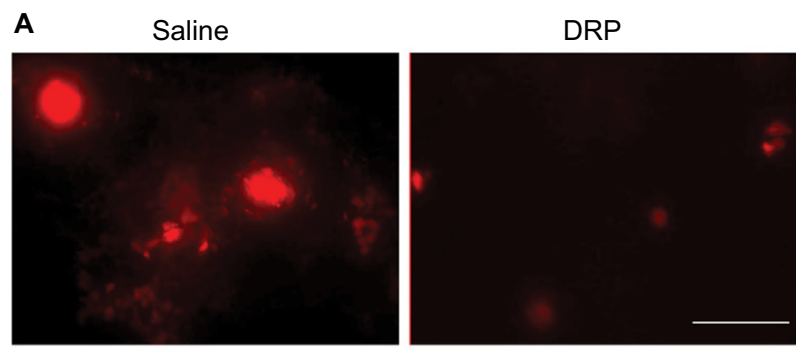

B

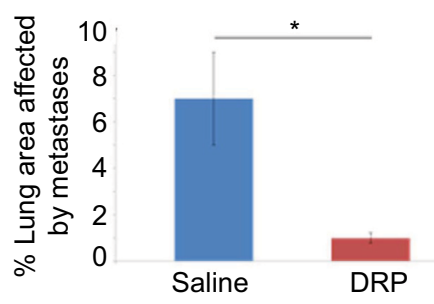

Figure 3 Effect of DRP on experimental metastasis of MDA-23I cells in lungs. Notes: (A) Representative fluorescence images ( $10 \times$ ) of lungs of mice 10 days after tail-vein injection of RFP-expressing MDA-23I cells. These mice were also injected with either saline or 2 ppm DRP three times over the course of the experiment. (B) A bar graph shows a comparison of the average \% lung area affected by metastases between control and DRP-treated mice (these data are based on analyses of 20 random $10 \times$ lung fields pooled from three mice in each group). A two-tailed Student's $t$-test was performed for testing statistical significance of the difference of the means between the two groups ( ${ }^{*} p<0.000$ I; scale bar: $\left.100 \mu \mathrm{m}\right)$

Abbreviations: DRP, drag-reducing polymer; RFP, red fluorescence protein.

cells that promote extravasations, for example, neutrophils and platelets, as shown previously. ${ }^{10}$ These possibilities will be better addressed by intravital imaging in the future studies.

Currently, clinically approved anticancer therapeutics are mostly targeted to inhibit growth of the primary tumor rather than blocking specific steps of metastasis. For those patients where vascular dissemination of tumor cells occurs very early, possibly even before a primary tumor is diagnosed, preventive strategies geared toward dissemination-promoting activities at the primary tumor sites should be minimally effective. However, in many other situations arresting tumor cells in the circulation offers an attractive opportunity for therapeutic intervention since it should not only prevent the metastatic seeding of CTC but may also enhance the efficacy of cytotoxic agents administered through systemic routes. ${ }^{21}$ Although DRPs were not formally tested for potential toxicity, many chronic animal studies demonstrated the absence of toxic effects after multiple injections over long periods of time. In general, since high molecular weight PEO is chemically neutral and no specific blood components can destroy these molecules, the major way of their elimination is mechanical degradation in blood circulation and excretion with urine, which involves the oxidation of the alcohol groups present on this polymer to a carboxylic acid. ${ }^{18}$

In conclusion, this is the very first, proof-of-concept study that demonstrated that hemodynamic/hemorheological modulation strategy can interfere with extravasation and metastatic seeding of tumor cells in the lungs. In the tailvein injection model, the injected tumor cells are mostly arrested in the lung vasculature and exclusively give rise to lung metastasis. Therefore, we were not able to assess whether there is any organ-tropism regarding the effect of DRP on the extravasation of cancer cells, and this is one of the limitations of this study (cardiac injection model that leads to multiple sites of metastasis may address this issue in the future). It will be also interesting to study the effect of DRP on the long-term fate of CTC. If future studies establish the generality of our findings extending to other types of cancer cells and other metastatic sites, it can open the possibility of a new line of adjuvant interventional strategy in cancer.

\section{Acknowledgments}

This study was supported by grants from the Congressionally Directed Medical Research Program of the Department of Defense (W81XWH-11-1-0122) and National Institute of Health (R01 CA108607) to PR.

\section{Disclosure}

The authors report no conflicts of interest in this work.

\section{References}

1. Joyce JA, Pollard JW. Microenvironmental regulation of metastasis. Nat Rev Cancer. 2009;9(4):239-252.

2. Huh SJ, Liang S, Sharma A, Dong C, Robertson GP. Transiently entrapped circulating tumor cells interact with neutrophils to facilitate lung metastasis development. Cancer Res. 2010;70(14):6071-6082.

3. Liang S, Slattery MJ, Wagner D, Simon SI, Dong C. Hydrodynamic shear rate regulates melanoma-leukocyte aggregation, melanoma adhesion to the endothelium, and subsequent extravasation. Ann Biomed Eng. 2008;36(4):661-671.

4. Liang S, Slattery MJ, Dong C. Shear stress and shear rate differentially affect the multi-step process of leukocyte-facilitated melanoma adhesion. Exp Cell Res. 2005;310(2):282-292.

5. Slattery MJ, Liang S, Dong C. Distinct role of hydrodynamic shear in leukocyte-facilitated tumor cell extravasation. Am J Physiol Cell Physiol. 2005;288(4):C831-C839.

6. Chiu JJ, Lee PL, Chen CN, et al. Shear stress increases ICAM-1 and decreases VCAM-1 and E-selectin expressions induced by tumor necrosis factor-[alpha] in endothelial cells. Arterioscler Thromb Vasc Biol. 2004;24(1):73-79.

7. Chiu JJ, Chen LJ, Lee PL, et al. Shear stress inhibits adhesion molecule expression in vascular endothelial cells induced by coculture with smooth muscle cells. Blood. 2003;101(7):2667-2674.

8. Fåhraeus R. The suspension stability of the blood. Physiol Rev. 1929;IX:241-75.

9. Kameneva MV, Wu ZJ, Uraysh A, et al. Blood soluble drag-reducing polymers prevent lethality from hemorrhagic shock in acute animal experiments. Biorheology. 2004;41(1):53-64.

10. Kameneva MV. Microrheological effects of drag-reducing polymers in vitro and in vivo. Int J Eng Sci. 2012;59:168-183.

11. Zhao R, Marhefka JN, Antaki JF, Kameneva MV. Drag-reducing polymers diminish near-wall concentration of platelets in microchannel blood flow. Biorheology. 2010;47(3-4):193-203. 
12. Marascalco PJ, Blair HC, Nieponice A, Robinson LJ, Kameneva MV Intravenous injections of soluble drag-reducing polymers reduce foreign body reaction to implants. ASAIO J. 2009;55(5):503-508.

13. Gau D, Ding Z, Baty C, Roy P. Fluorescence resonance energy transfer (FRET)-based detection of profilin-VASP interaction. Cell Mol Bioeng. 2011;4(1):1-8.

14. Ding Z, Joy M, Bhargava R, et al. Profilin-1 downregulation has contrasting effects on early vs late steps of breast cancer metastasis. Oncogene. 2014;33(16):2065-2074.

15. Sato H, Suzuki M. Deformability and viability of tumor cells by transcapillary passage, with references to organ affinity of Metastasis in cancer. In: Weiss, L, editor. Fundamental Aspects of Metastasis. North-Holland, Amsterdam; 1976:311-317.

16. Weiss L, Dimitrov DS. A fluid mechanical analysis of the velocity, adhesion, and destruction of cancer cells in capillaries during metastasis. Cell Biophys. 1984;6(1):9-22.
17. Munn DH, Cheung NK. Antibody-independent phagocytosis of tumor cells by human monocyte-derived macrophages cultured in recombinant macrophage colony-stimulating factor. Cancer Immunol Immunother. 1995;41(1):46-52.

18. Chernysheva G, Smol'yakova V, Plotnikov M, Cherkashina I. Pharmacokinetics of high molecular weight polyethylene oxide. Pharm Chem J. 2006;40(9):467-468.

19. Mostardi RA, Thomas LC, Greene HL, VanEssen F, Nokes RF. Suppression of atherosclerosis in rabbits using drag reducing polymers Biorheology. 1978;15(1):1-14.

20. Faruqui FI, Otten MD, Polimeni PI. Protection against atherogenesis with the polymer drag-reducing agent separan AP-30. Circulation. 1987;75(3):627-635.

21. Labelle M, Hynes RO. The initial hours of metastasis: the importance of cooperative host-tumor cell interactions during hematogenous dissemination. Cancer Discov. 2012;2(12):1091-1099.
Breast Cancer - Targets and Therapy

\section{Publish your work in this journal}

Breast Cancer - Targets and Therapy is an international, peerreviewed open access journal focusing on breast cancer research, identification of therapeutic targets and the optimal use of preventative and integrated treatment interventions to achieve improved outcomes, enhanced survival and quality of life for the cancer patient

\section{Dovepress}

The manuscript management system is completely online and includes a very quick and fair peer-review system, which is all easy to use. Visit http://www.dovepress.com/testimonials.php to read real quotes from published authors. 